\title{
Synergistic inhibitory effects of the oxyresveratrol and dacarbazine combination against melanoma cells
}

\author{
SANG GYU LEE ${ }^{1}$, DONG GUN LEE ${ }^{2}$, YONG HOON JOO ${ }^{2}$ and NAMHYUN CHUNG ${ }^{1}$ \\ Departments of ${ }^{1}$ Biotechnology and ${ }^{2}$ Biosystems and Biotechnology, College of Life Sciences \\ and Biotechnology, Korea University, Seoul 02841, Republic of Korea
}

Received January 20, 2021; Accepted May 4, 2021

DOI: $10.3892 / 01.2021 .12928$

\begin{abstract}
Various therapies have been developed to target malignant melanoma, which is associated with a high mortality rate worldwide. Although dacarbazine (DTIC) is employed for treating melanoma, it is associated with several side effects. Hence, patients with melanoma are co-treated with additional drugs to mitigate the side effects of DTIC. In the present study, synergistic therapeutic effects of the DTIC/oxyresveratrol (ORT) combination were examined using the human malignant melanoma WM-266-4 cell line. Treatment with ORT and DTIC inhibited the proliferation of WM-266-4 cells. Compared with those in the ORT- and DTIC-treated groups, the proportion of cells arrested at the S phase, as well as apoptotic rates, were increased in the ORT and DTIC co-treatment group. In WM-266-4 cells, synergistic proliferation-inhibitory activities of the ORT/DTIC combination were assessed based on cell viability and migration, antioxidant capacity, cytokine production, cell cycle arrest, apoptotic rate and protein expression through WST-1 assay, wound healing assay, flow cytometry and western blotting. Furthermore, the expression levels of proteins, including NOTCH, involved in the pathogenesis of solid cancers, such as melanoma, were examined. Overall, the ORT/DTIC combination synergistically promoted cell cycle arrest at the S phase and the apoptosis of WM-266-4 cells. Thus, this combination treatment may serve as a novel therapeutic strategy for treating malignant melanoma.
\end{abstract}

Correspondence to: Professor Namhyun Chung, Department of Biotechnology, College of Life Sciences and Biotechnology, Korea University, 145 Anam-ro, Seongbuk, Seoul 02841, Republic of Korea E-mail: nchung@korea.ac.kr

Abbreviations: cDNA, complementary DNA; DMEM, Dulbecco's modified Eagle's medium; DPPH, 2,2-diphenyl-1-picryl-hydrazyl-hyd rate; DTIC, dacarbazine; IC $_{50}$, half-maximal inhibitory concentration; IL, interleukin; LPS, lipopolysaccharide; ORT, oxyresveratrol; PI, propidium iodide; RT-qPCR, reverse transcription-quantitative PCR; TNF- $\alpha$, tumor necrosis factor- $\alpha$

Key words: chemical drug, synergistic effect, Notch signaling, stilbenoid, $\mathrm{S}$ phase arrest

\section{Introduction}

Melanoma is one of the leading causes of cancer-related deaths worldwide (1). In 2020, approximately 100,350 new melanoma cases were expected in the United States of America (2), indicating that melanoma incidence would increase by approximately $35.8 \%$ in 2020 compared with that in 2015 (3). Accordingly, there has been a global increase in the incidence of melanoma. Patients with melanoma exhibit resistance to cancer therapy. Therefore, there is an urgent need to develop novel therapeutics and treatment strategies against this disease (4). Notably, malignant melanoma accounts for $65 \%$ of deaths caused by various types of melanoma; however, early diagnosis could improve therapeutic outcomes. Dacarbazine (DTIC) is used for treating melanoma. However, clinical studies have reported that DTIC administration can result in various side effects such as vomiting, nausea, and anorexia (5). In the last few decades, DTIC has been widely used to treat patients with melanoma, despite observed side effects. Accordingly, DTIC is frequently co-administered with other anticancer drugs or immunotherapeutics to alleviate these side effects (6). Moreover, a previous study has reported the co-administration of DTIC and saponin, a substance of natural origin, in an attempt to reduce the DTIC dose (7). Although several hypotheses have been proposed, the anticancer mechanism of DTIC needs to be comprehensively elucidated (6).

Oxyresveratrol (ORT), a naturally occurring stilbenoid, was first isolated from the heartwood of Artocarpus lakoocha, a monkey fruit in tropical regions (8). Previous studies have demonstrated the proliferation-inhibitory activity of ORT against stomach and ovarian cancers (9). Reportedly, the enhanced tyrosinase activity associated with melanoma is mitigated following treatment with ORT $(10,11)$. Furthermore, the antioxidative capacity of ORT is two-fold higher than that of resveratrol (12). ORT consumption may exert beneficial effects on human health, as circulating ORT has an absorption rate of more than $50 \%$ in tissues (13).

It is well known that malignant melanoma can develop resistance to chemotherapy. Hence, various reports have recommended administering additional anticancer drugs in combination with DTIC, for malignant melanoma (14-17). However, therapeutic efficacy of the ORT/DTIC combination in melanoma has not been previously reported. In the present 
study, we examined synergistic proliferation-inhibitory effect of the ORT/DTIC combination against a malignant melanoma cell line.

\section{Materials and methods}

Cell culture. The human malignant melanoma cell line WM-266-4 and the mouse macrophage cell line RAW264.7 were procured from the Korean Cell Line Bank. The cells were cultured in Dulbecco's modified Eagle's medium (DMEM) supplemented with $100 \mathrm{U} / \mathrm{ml}$ penicillin, $100 \mu \mathrm{g} / \mathrm{ml}$ streptomycin, and $10 \%$ heat-inactivated fetal bovine serum. The culture reagents used in this study were purchased from Welgene. The cell lines were subcultured in $100-\mathrm{mm}$ cell culture plates (Sarstedt), with fresh DMEM replaced every three days. All cells were cultured at $37^{\circ} \mathrm{C}$ in a humidified atmosphere containing $5 \% \mathrm{CO}_{2}$.

Cell viability. Cell viability was measured using the WST-1 assay (Takara Bio). In brief, WM-266- 4 cells $\left(5 \times 10^{4}\right.$ cells/well) were cultured in 96-well cell culture plates (SPL Life Science) and incubated at $37^{\circ} \mathrm{C}$ for $3 \mathrm{~h}$ in a humidified atmosphere containing $5 \% \mathrm{CO}_{2}$. Next, the cells were treated with ORT (50-170 $\mu \mathrm{M}$; Sigma-Aldrich; Merck KGaA; ORT-treated group), DTIC (0.5-2.5 mM; Tokyo Clinic Industry; DTIC-treated group), or an ORT (70 $\mu \mathrm{M}) / \mathrm{DTIC}(0.5-2.5 \mathrm{mM})$ combination (co-treatment group) for $48 \mathrm{~h}$. After $48 \mathrm{~h}$, each well was washed twice with phosphate-buffered saline (PBS) and $10 \mu \mathrm{l}$ WST-1 reagent was added to $90 \mu \mathrm{l}$ DMEM. After $2 \mathrm{~h}$, the absorbance of the mixture was measured at $450 \mathrm{~nm}$ using a microplate reader (EL800; Bio-Tek).

Radical scavenging activity. The antioxidant potential of ORT was determined using the 2,2-diphenyl-1-picryl-hydrazyl-hydrate (DPPH; Sigma-Aldrich; Merck KGaA) assay, following a previously described method with minor modifications (18). In brief, $150 \mu \mathrm{l}$ of DPPH solution $(100 \mu \mathrm{M})$, dissolved in $100 \%$ ethanol, was added to each well of a 96-well plate, followed by the addition of $50 \mu \mathrm{l}$ of the half-maximal inhibitory concentration $\left(\mathrm{IC}_{50}\right)$ of ORT, DTIC, or the ORT/DTIC combination. For the control group, $50 \mu \mathrm{l}$ of $14.04 \mathrm{M}$ dimethyl sulfoxide and/or $1 \mathrm{M} \mathrm{HCl}$ was added instead of drugs. The absorbance of the reaction mixture was measured at $520 \mathrm{~nm}$, using a microplate reader (BioTek EL800), after incubation for $10 \mathrm{~min}$. The DPPH radical scavenging activity was calculated as follows: DPPH radical scavenging activity $(\%)=[(1-(\mathrm{A} / \mathrm{B})] \times 100$, where A and B are the absorbance values of the treatment and control groups, respectively.

Measurement of cytokine concentrations. In brief, RAW264.7 cells were treated with lipopolysaccharide (LPS; Sigma-Aldrich; Merck KGaA) to induce cytokine expression. Next, the cells were immediately treated with the $\mathrm{IC}_{50}$ of ORT, DTIC, or the ORT/DTIC combination for $48 \mathrm{~h}$. The culture medium was collected to examine interleukin (IL)-6 (DY406; R\&D Systems) and tumor necrosis factor (TNF)- $\alpha$ (DY410; R\&D Systems) levels using enzyme-linked immunosorbent assay kits (R\&D Systems) according to the manufacturer's instructions.

Wound healing assay. Cell migration was analyzed using the CytoSelect 24-well wound healing assay (Cell Biolabs). First, square blocks were inserted into each well of a 24-well plate. WM-266-4 cells $\left(5 \times 10^{5}\right.$ cells/well) were seeded in 24-well plates, and the cells were cultured for $24 \mathrm{~h}$. The cells were washed twice with PBS (pH 7.4) and treated with the $\mathrm{IC}_{50}$ of ORT, DTIC, or the ORT/DTIC combination. After $24 \mathrm{~h}$, cell migration to the wound area was comparatively analyzed using a microscope (CK40; Olympus).

Cell cycle analysis. In brief, WM-266-4 cells seeded onto $100-\mathrm{mm}$ cell culture plates were treated with the $\mathrm{IC}_{50}$ of ORT, DTIC, or the ORT/DTIC combination for $24 \mathrm{~h}$. The cells were harvested and fixed with $70 \%(\mathrm{w} / \mathrm{v})$ ethanol at $20^{\circ} \mathrm{C}$ for at least $4 \mathrm{~h}$. The fixed cells were washed thrice with PBS and treated with a propidium iodide (PI; Sigma-Aldrich; Merck KGaA) solution $(50 \mu \mathrm{g} / \mathrm{ml}$ PI, $0.1 \mathrm{mg} / \mathrm{ml}$ RNase [Sigma-Aldrich; Merck KGaA], and 0.1\% Triton X-100 [v/v; Sigma-Aldrich; Merck KGaA] in PBS). Then, the treated cells were incubated at $25^{\circ} \mathrm{C}$ for $30 \mathrm{~min}$ in the dark and analyzed using a flow cytometer (FACSCalibur Cell Analyzer; BD Biosciences). The data were analyzed using the ModFit Lt software (version 4.0; Verity Software House, ME, USA).

Analysis of apoptosis. After seeding onto 100-mm cell culture plates, WM-266-4 cells were treated with the $\mathrm{IC}_{50}$ of ORT, DTIC, or the ORT/DTIC combination for $48 \mathrm{~h}$ and then transferred into a conical tube. The cells were then washed thrice with PBS and suspended in $200 \mu 1$ of Annexin V binding buffer, a component of the apoptosis detection kit (BD Biosciences). The cells were incubated with $5 \mu \mathrm{l}$ PI and $5 \mu 1$ fluorescein isothiocyanate-conjugated Annexin V for $20 \mathrm{~min}$ at room temperature in the dark. Apoptosis was measured using a flow cytometer (FACSCalibur Cell Analyzer). The fluorescence intensities of PI and Annexin V-FITC were analyzed at excitation/emission wavelengths of $488 / 617 \mathrm{~nm}$ and $488 / 530 \mathrm{~nm}$, respectively.

Reverse transcription-quantitative PCR (RT- $q P C R)$. Total RNA was extracted from untreated WM-266-4 cells and WM 266-4 cells treated with the $\mathrm{IC}_{50}$ of ORT, DTIC, or the ORT/DTIC combination, using the TRIzol reagent (Invitrogen; Thermo Fisher Scientific, Inc.). Complementary DNA (cDNA) was synthesized from total RNA using a first-strand synthesis kit (GeneAll). The cDNA concentration was determined using a NanoDrop spectrophotometer (Coliri LB 915; JC Bio). qRT-PCR was performed using CFX Connect Real-Time PCR (Bio-Rad) and BrightGreen 2X qPCR MasterMix (ABM, Vancouver, Canada), with 50 amplification cycles. In the present study, the expression levels of $\mathrm{NOTCH} 2, \mathrm{NOTCH} 3$, $M Y C, C C N D 1, B A X, C A S P 3$ and $C A S P 9$ were comparatively analyzed among different treatment groups. The primers used in the qRT-PCR analysis are listed in Table I. The expression levels of target genes were normalized to those of GAPDH (19-24). Relative gene expression levels were calculated using the $2^{-\Delta \Delta C t}$ method (25).

Western blotting. Proteins were extracted from WM-266-4 cells treated with the $\mathrm{IC}_{50}$ of ORT, DTIC, or the ORT/DTIC combination, using the M-PER mammalian protein extraction reagent (Thermo Fisher Scientific, Inc.). Protein concentration was determined using a bicinchoninic acid protein assay kit 
Table I. Primer sequences used for reverse transcription-quantitative PCR.

\begin{tabular}{lll}
\hline Gene & \multicolumn{1}{c}{ Forward primer $\left(5^{\prime}-3^{\prime}\right)$} & \multicolumn{1}{c}{ Reverse primer $\left(5^{\prime}-3^{\prime}\right)$} \\
\hline NOTCH2 & CGTTTAGTCAGGAATATGCGG & GGACACATTTATGTACCCAGAG \\
NOTCH3 & GCTTCTCAGGTCCTCGCTGT & GGCACAGTGACAGGTGAAGG \\
MYC & CCAGCAGCGACTCTGAGG & CCAAGACGTTGTGTGTTC \\
CCND1 & CGATGCCAACCTCCTCAACGA & TCGCAGACCTCCAGCATCCA \\
BAX & GGATGCGTCCACCAAGAAG & GCCTTGAGCACCAGTTTGC \\
CASP3 & ATGGTTTGAGCCTGAGCAGA & GGCAGCATCATCCACACATAC \\
CASP9 & GCAGGCTCTGGATCTCGGC & GCTGCTTGCCTGTTAGTCGC \\
GAPDH & CGGAGTCAACGGATTTGGTCGTAT & AGCCTTCTCCATGGTGGTGAAGAC
\end{tabular}

CCND1, cyclin D1; CASP, caspase.

(Thermo Fisher Scientific, Inc.). For each group, $25 \mu \mathrm{g}$ of total protein was subjected to sodium dodecyl sulfate-polyacrylamide gel electrophoresis using a $10-12 \%$ gel. Next, the resolved proteins were transferred onto polyvinylidene fluoride membranes $(0.45 \mu \mathrm{m}$; EMD Millipore). The membranes were blocked with $5 \%$ skim milk or $5 \%$ bovine serum albumin and incubated overnight at $4^{\circ} \mathrm{C}$ with anti- $\beta$-actin, anti-BAX, anti-CASP3, anti-CASP9, anti-MYC, anti-CCND1, anti-NOTCH2 and anti-NOTCH3 antibodies diluted 1,000-fold (Cell Signaling Technology). Then, the membranes were probed with secondary antibodies, diluted 1,000-fold, for $2 \mathrm{~h}$ at room temperature. The antigen-antibody complexes were visualized using enhanced chemiluminescence western blotting detection reagents (Thermo Fisher Scientific, Inc.). ChemiDoc XRS (Bio-Rad) and the Image Lab software (version 4.1; Bio-Rad) were used to analyze the blot images.

Statistical analysis. All results of the present study were analyzed using analysis of variance at a 5\% significance level. Data were treated by one-way ANOVA and Tukey's honestly significant difference test (Tukey's HSD test). ANOVA was performed using SPSS version 20.0 (IBM Corp.). In the present study, all experiments were performed at least thrice.

\section{Results}

The dose-dependent effects of ORT, DTIC, and the ORT/DTIC combination on cell viability are shown in Fig. 1. ORT and DTIC decreased cell viability in a dose-dependent manner. Compared with that of the control cells, the percentage viability of cells treated with $50,80,110,140$ and $170 \mu \mathrm{M}$ ORT was approximately 92.9, 77.9, 60.6, 48.7 and 34.2\%, respectively. Compared with that of the control cells, the percentage viability of cells treated with $0.5,1,1.5,2$ and $2.5 \mathrm{mM}$ DTIC was approximately 73.6, 53.0, 37.6, 30.9 and $25.6 \%$, respectively. The $\mathrm{IC}_{50}$ values of ORT and DTIC were approximately $140 \mu \mathrm{M}$ and $1 \mathrm{mM}$, respectively. The cells were co-treated with $70 \mu \mathrm{M}$ ORT and various DTIC concentrations (0.25-1.0 mM) (Fig. 1C). The percentage viability of cells co-treated with $0.25,0.50,0.75$ and $1.0 \mathrm{mM}$ DTIC was $52.9,48.0,41.4$ and $34.6 \%$, respectively, when compared with that of the control cells. This indicated that DTIC decreased cell viability in a dose-dependent manner. In particular, the viability of cells co-treated with $70 \mu \mathrm{M}$ ORT and $0.25 \mathrm{mM}$ DTIC was $52.9 \%$. Thus, the $\mathrm{IC}_{50}$ of the ORT/DTIC combination was approximately two-fold lower than that of ORT and four-fold lower than that of DTIC.

Flow cytometry was performed to examine the effects of ORT, DTIC, and the ORT/DTIC combination on the cell cycle (Fig. 2). The proportion of WM-266-4 cells arrested in the $\mathrm{S}$ phase was examined in the different treatment groups (Fig. 2A). The analysis revealed that the cell proportions arrested at the $\mathrm{S}$ phase in the control, ORT-treated, DTIC-treated, and co-treatment groups were $17.00 \pm 0.74,45.93 \pm 0.65,26.13 \pm 0.87$ and $60.22 \pm 3.58 \%$, respectively (Fig. 2B). This suggested that ORT and DTIC synergistically induced cell cycle arrest following co-administration.

Next, flow cytometry with Annexin V/PI double staining was employed to assess apoptosis in WM-266-4 cells. Apoptotic cells were classified as follows: early apoptotic cells (Annexin $\mathrm{V}^{+} / \mathrm{PI}^{-}$) and late apoptotic cells (Annexin $\mathrm{V}^{+} / \mathrm{PI}^{+}$). The pattern of apoptosis varied between the control and treatment groups (Fig. 3A). The proportion of cells exhibiting total apoptosis (early apoptosis plus late apoptosis) in the control, ORT-treated, DTIC-treated, and co-treatment groups was $5.94 \pm 0.03,15.06 \pm 1.34,10.77 \pm 1.00$ and $23.94 \pm 3.98 \%$, respectively (Fig. 3B). Compared with that in the control group, the proportion of apoptotic cells was increased in the ORT- and DTIC-treated groups. Additionally, the proportion of apoptotic cells was further increased in the co-treatment groups when compared with that in the ORT- and DTIC-treated groups, indicating that ORT and DTIC synergistically induced apoptosis in WM-266-4 cells.

Next, the mRNA expression levels of genes related to the cell cycle and apoptosis were measured using qRT-PCR (Fig. 4). Among genes associated with the $\mathrm{S}$ phase, the relative mRNA expression levels of $\mathrm{NOTCH} 2, \mathrm{NOTCH} 3$, and $\mathrm{CCND} 1$ varied in the ORT-treated group when compared with those in the control group. The expression levels of NOTCH2, NOTCH3, and $C C N D 1$ were markedly higher in the DTIC-treated group than in the control group. However, the expression levels of these genes in the co-treatment group were similar to those observed in the control group (Fig. 4). The expression pattern of MYC markedly differed from that of the three genes. Compared with that in the control group, the expression of 

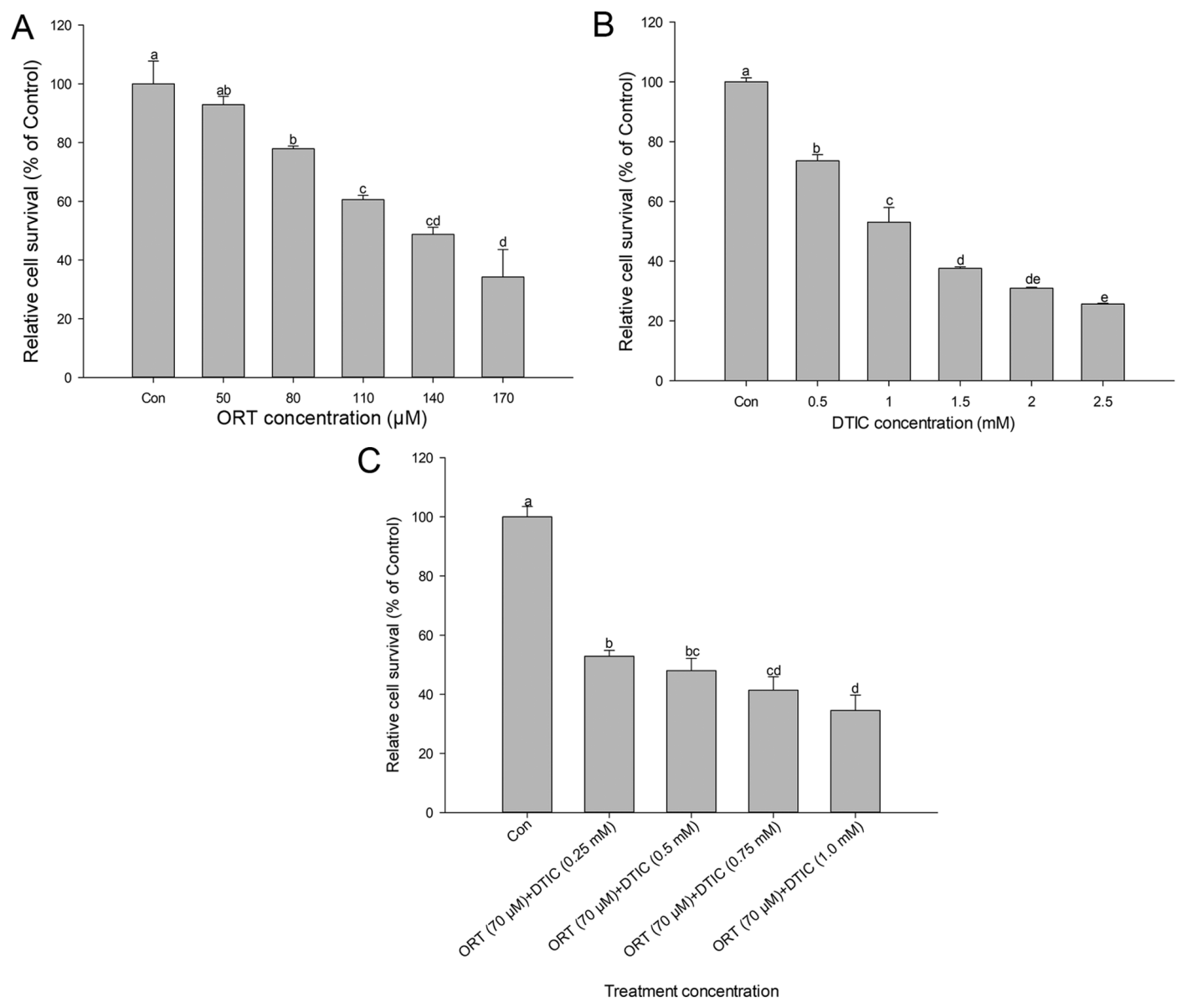

Figure 1. Dose-dependent effects of ORT and DTIC on cell viability. WM-266-4 cells were treated with (A) 50-170 $\mu \mathrm{M}$ ORT, (B) $0.5-2.5 \mathrm{mM}$ DTIC and (C) a combination of $70 \mu \mathrm{M}$ ORT and $0.25-1.0 \mathrm{mM}$ DTIC. A dose-dependent decrease in cell viability was observed in the treatment groups. Half-maximal inhibitory concentration values were based on relative cell survival. Values followed by the same small letter are not significantly different $(\mathrm{P}<0.05)$. ORT, oxyresveratrol; DTIC, dacarbazine; Con, control.

$M Y C$ was increased in the ORT-treated group. However, $M Y C$ expression in the DTIC-treated and co-treatment groups was approximately seven-fold higher than that in the control group (Fig. 4). On analyzing the expression levels of CASP3 and CASP9 (apoptosis-related genes), no specific pattern was observed. Compared with those in the control group, BAX expression levels were markedly decreased in the ORT-treated, DTIC-treated, and co-treatment groups, with the lowest levels observed in the co-treatment group. Compared with those in the control group, the expression levels of CASP3 were markedly reduced in the ORT-treated group and considerably elevated in the DTIC-treated group. The expression levels of $C A S P 3$ in the co-treatment groups were similar to those in the control group; however, the expression levels of CASP 9 were upregulated by approximately 2.5 -fold in the co-treatment group as compared to those in the control group (Fig. 4).

The effects of ORT, DTIC, and the ORT/DTIC combination on the expression levels of NOTCH2, NOTCH3, and BAX were examined using western blotting. Compared with those in the control, ORT-treated, and DTIC-treated groups, the expression levels of $\mathrm{NOTCH} 2$, NOTCH3, and BAX were markedly reduced in the co-treatment group (Fig. 5A and B). The expression level of CCND1 was markedly higher in the co-treatment group than in the ORT- and DTIC-treated groups. Furthermore, the expression level of MYC in the ORT-treated, DTIC-treated, and co-treatment groups was higher than that in the control group. Compared with that in the DTIC-treated group, the expression level of MYC was increased in the ORT-treated and co-treatment groups. In the ORT-treated, DTIC-treated, and co-treatment groups, the expression levels of CASP3 and CASP9 were higher than those in the control group.

\section{Discussion}

In the present study, the WST-1 assay was used to examine the effects of ORT, DTIC, or the ORT/DTIC combination on WM-266-4 melanoma cell viability. This assay enables the measurement of intact cell viability. The $\mathrm{IC}_{50}$ of the ORT, DTIC, or ORT/DTIC combination was estimated using the WST-1 assay. The viability of WM-266-4 cells decreased to $50 \%$ following treatment with $140 \mu \mathrm{M}$ ORT, $1 \mathrm{mM}$ DTIC, or the $70 \mu \mathrm{M}$ ORT/0.25 mM DTIC combination (Fig. 1). This indicated that the concentration of DTIC, an anticancer drug, required to suppress the proliferation of WM-266-4 cells by $50 \%$ decreased from $1 \mathrm{mM}$ to $0.25 \mathrm{mM}$ in the presence of $70 \mu \mathrm{M}$ ORT. The cytotoxicity of ORT, an antioxidant, is reportedly lower than that of DTIC (26). Moreover, ORT is not cytotoxic in normal cells, such as human keratinocyte and mouse melanocyte cells, at relatively high concentrations $(27,28)$. 
A
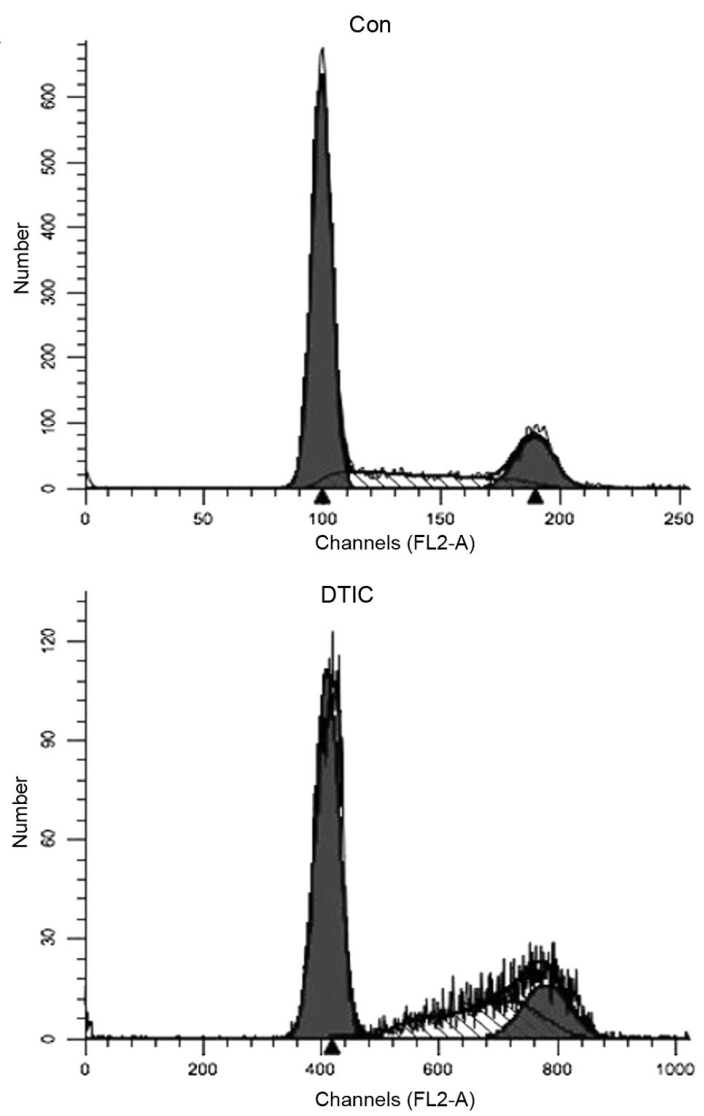
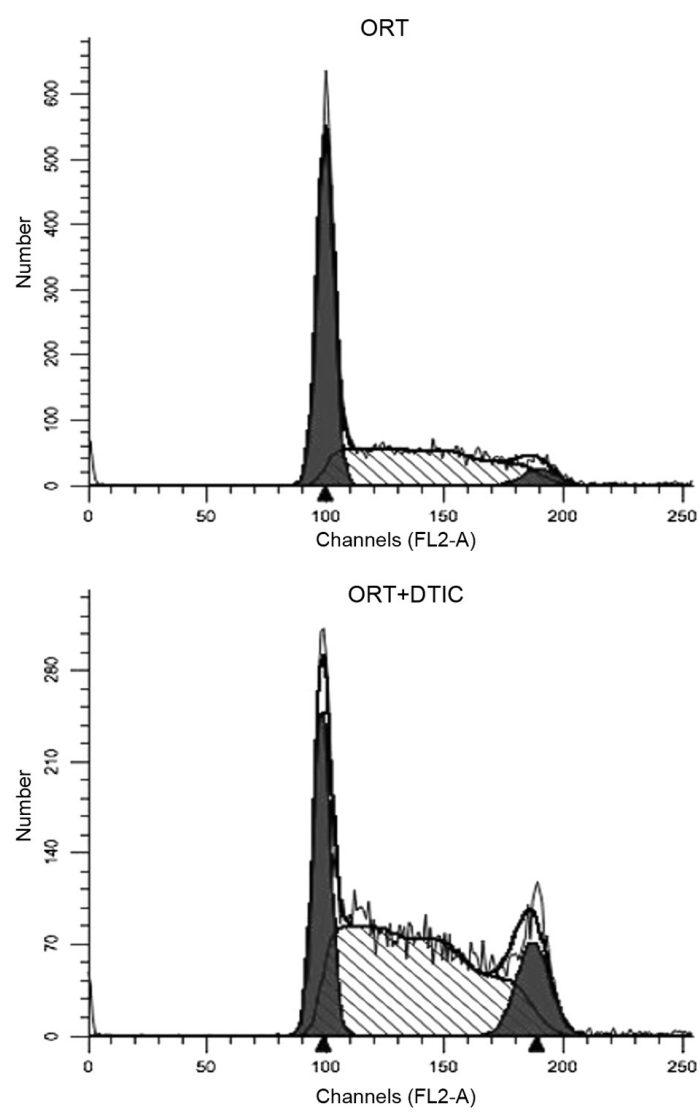

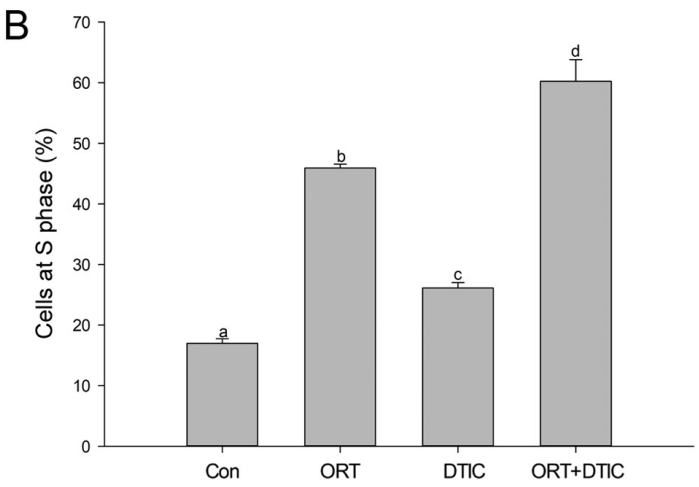

Figure 2. Cell cycle analyses of the Con (untreated), ORT-treated, DTIC-treated and ORT+DTIC co-treatment groups. (A) Histograms of flow cytometric data. (B) Proportion of WM-266-4 cells arrested at the S phase. Values followed by the same small letter are not significantly different (P<0.05). ORT, oxyresveratrol; DTIC, dacarbazine; Con, control.

The DPPH radical scavenging activity assay is widely utilized to measure the antioxidant potential of chemicals (29). ORT, a stilbenoid and aglycone of mulberroside A, has four hydroxyl groups on the benzene ring. The structural characteristics of ORT indicate that it is a potent antioxidant. ORT is frequently employed to alleviate oxidative stress, which is a common cause of cellular dysfunction, injury, and death (30). As shown in Fig. S1, the antioxidant capacities of ORT and DTIC were approximately $12.55 \pm 0.003$ and $1.26 \pm 0.005 \%$, respectively, thus indicating the potent antioxidant capacity of ORT. The antioxidant capacity of ORT $(12.55 \pm 0.003 \%)$ was lower than that of the ORT/DTIC combination $(16.18 \pm 0.01 \%)$. This suggests that the ORT/DTIC combination exhibited synergistic antioxidant activities.

IL-6, which is involved in T cell differentiation and proliferation, mediates various functions, including vital pro-inflammatory functions in response to infection or injury. However, excessive production of IL- 6 contributes to chronic inflammation, which leads to various diseases, including chronic arthritis and osteoporosis (31-33). TNF- $\alpha$ plays an important role in the innate immune response against LPS (34). Reportedly, ORT mitigates the LPS-induced increase in TNF- $\alpha$ levels (35). The effects of ORT and DTIC on the production of IL- 6 and TNF- $\alpha$ were evaluated by treating RAW264.7 cells with the $\mathrm{IC}_{50}$ of ORT, DTIC, or the ORT/DTIC combination. As shown in Fig. S2A, the lowest IL-6 concentration was observed following ORT treatment $(0.33 \pm 0.08 \mathrm{ng} / \mathrm{ml})$. Treatment with DTIC increased IL-6 concentration $(3.76 \pm 0.71 \mathrm{ng} / \mathrm{ml})$. However, co-treatment with ORT and DTIC decreased the concentration of IL- 6 to $0.69 \pm 0.06 \mathrm{ng} / \mathrm{ml}$, which was marginally higher than that in the ORT-treated group (Fig. S2A). These results demonstrate 


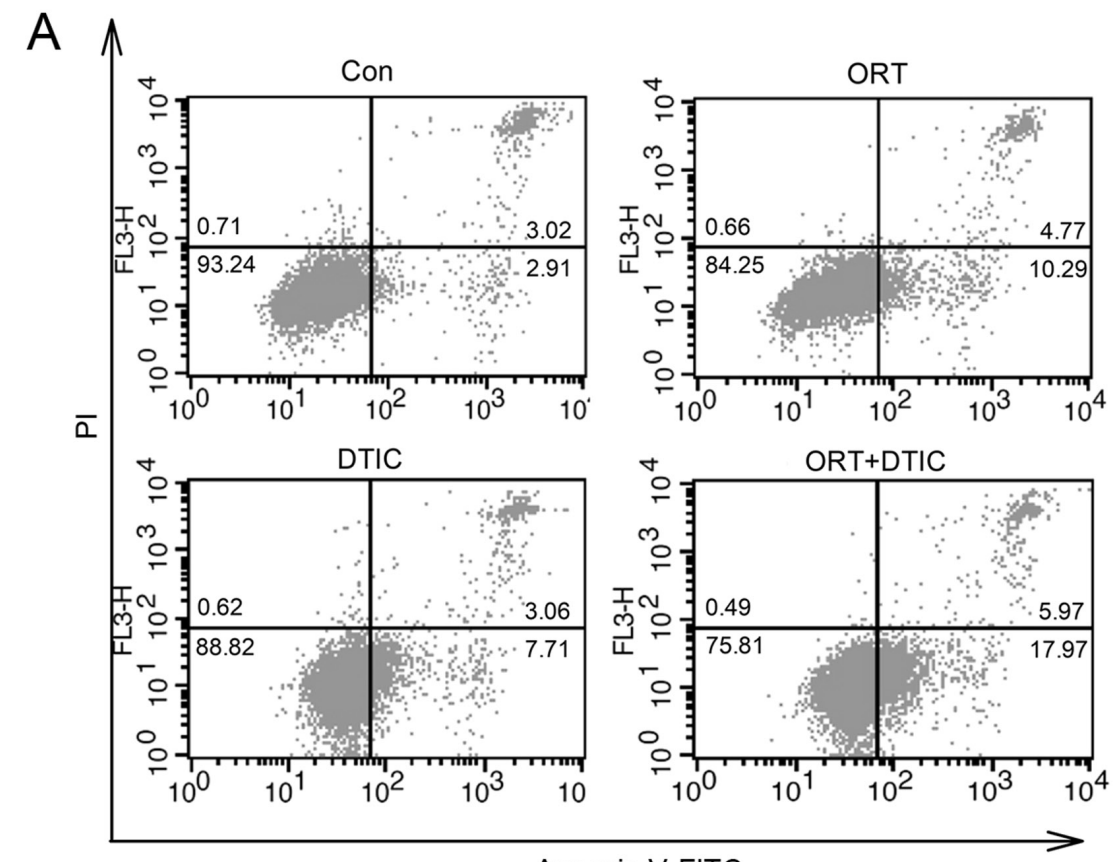

Annexin V-FITC

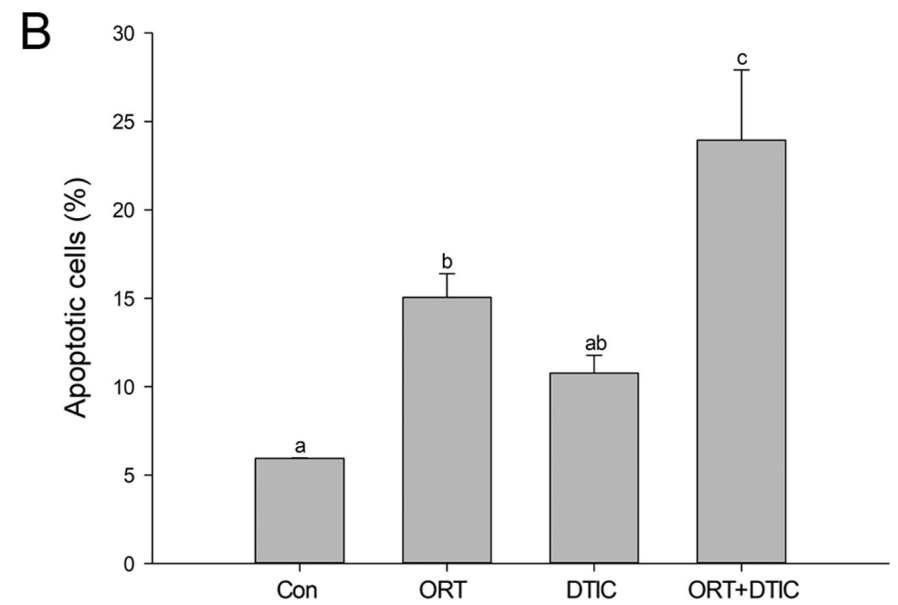

Figure 3. Flow cytometric analyses of apoptosis in the Con (untreated), ORT-treated, DTIC-treated and ORT+DTIC co-treatment groups. (A) WM-266-4 cells were stained with Annexin V/PI and analyzed by flow cytometry. (B) Percentage of apoptotic cells, including early apoptotic (Annexin $\left.\mathrm{V}^{+} / \mathrm{PI}\right)$ and late apoptotic cells (Annexin $\mathrm{V}^{+} / \mathrm{PI}^{+}$). Values followed by the same small letter are not significantly different $(\mathrm{P}<0.05)$. ORT, oxyresveratrol; DTIC, dacarbazine; Con, control; PI, propidium iodide.

that ORT suppresses the DTIC-induced increase in IL-6 levels, indicating that the ORT/DTIC combination exerts a synergistic inhibitory effect on IL-6 production. As shown in Fig. S2B, the concentration of TNF- $\alpha$ in the ORT- and DTIC-treated groups was lower than that in the control group. The concentration of TNF- $\alpha$ was the lowest in the co-treatment group. These findings indicate that co-treatment with ORT and DTIC can synergistically mitigate LPS-induced IL- 6 and TNF- $\alpha$ production. Previous studies have revealed that TNF- $\alpha$ and IL- 6 are involved in the mitogen-activated protein kinase (MAPK) pathway. Thus, co-treatment with ORT and DTIC may modulate the MAPK pathway (36). The levels of IL- 6 and TNF- $\alpha$ were downregulated in RAW264.7 cells, suggesting that the combination of ORT and DTIC could exert synergistic effects in vitro and in vivo, which might allow the coexistence of cancer and immune cells.

The analysis of cell cycle arrest is an important tool for examining the effects of chemicals on the cell cycle (37). DTIC has been reported to induce $S$ phase cell cycle arrest (38). As shown in Fig. 2, the proportion of cells arrested during the $\mathrm{S}$ phase was higher in the co-treatment group $(60.22 \pm 3.58 \%)$ than in the ORT-treated $(45.93 \pm 0.65 \%)$ and DTIC-treated groups $(26.13 \pm$ $0.87 \%$ ). Thus, the proportion of cells arrested in the $\mathrm{S}$ phase in the co-treatment group was two-fold higher than that in the DTIC-treated group (Fig. 2). S phase arrest correlates with the expression of various proteins. For example, upregulated expression of CCND1 is associated with S phase arrest (39). Another study has reported that MYC overexpression promotes $\mathrm{S}$ phase arrest (40). Additionally, the downregulated expression of NOTCH2 and NOTCH3 and upregulated expression of CCND1 and MYC reportedly reduce cell migration ability (41). As shown in Fig. 5, western blotting revealed that the expression of NOTCH2 and NOTCH3 was downregulated in the co-treatment group. Additionally, the expression levels of CCND1 and MYC were higher in the co-treatment group than in the control group. The expression level of MYC in the ORT-treated group 

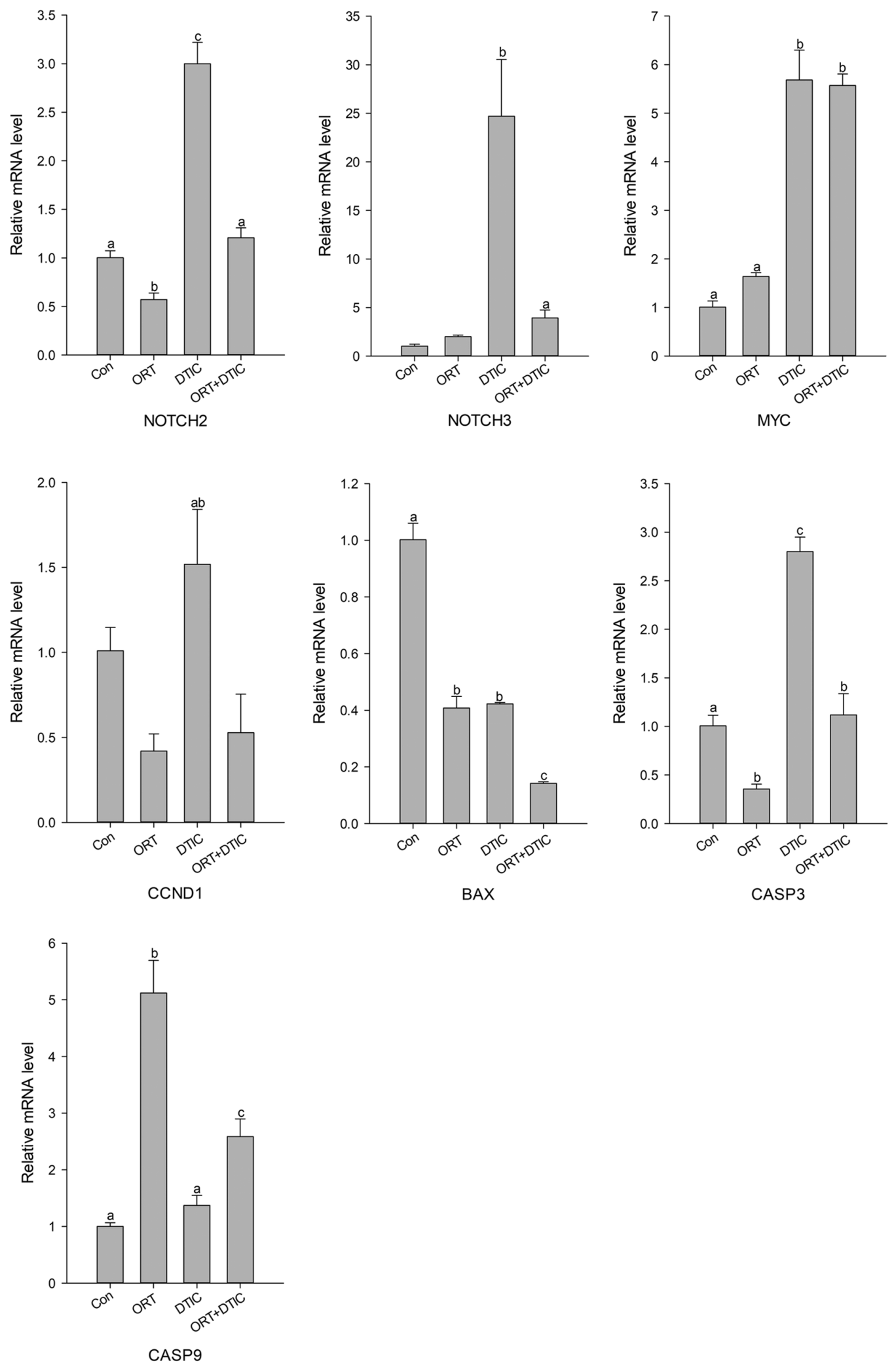

Figure 4. mRNA expression levels of seven genes in the Con (untreated), ORT-treated, DTIC-treated and ORT+DTIC co-treatment groups. The relative mRNA expression levels of NOTCH2, NOTCH3, MYC, CCND1, BAX, CASP9 and CASP3 were analyzed using reverse transcription-quantitative PCR. Values followed by the same small letter are not significantly different $(\mathrm{P}<0.05)$. ORT, oxyresveratrol; DTIC, dacarbazine; Con, control; CCND1, cyclin D1; CASP, caspase.

was similar to that in the co-treatment group, indicating that ORT promoted cell cycle arrest at the $\mathrm{S}$ phase by upregulating MYC expression. The expression level of BAX was lower in the co-treatment group than in the ORT- and DTIC-treated groups. However, the expression levels of CASP3 and CASP9 did not markedly differ among the three treatment groups. Compared with those in the control group, the protein expression levels of CASP3 and CASP9 were increased in the three treatment groups. The qRT-PCR results were consistent with western blotting results in some cases, while varying in others (Fig. 4). 
A

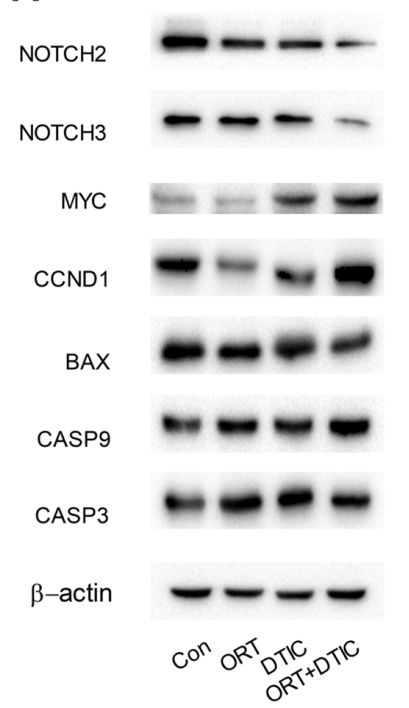

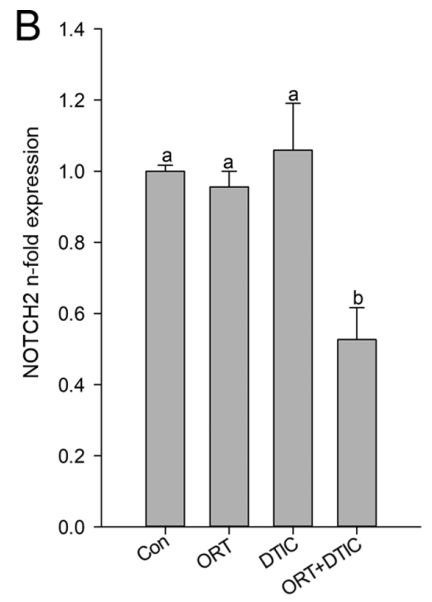
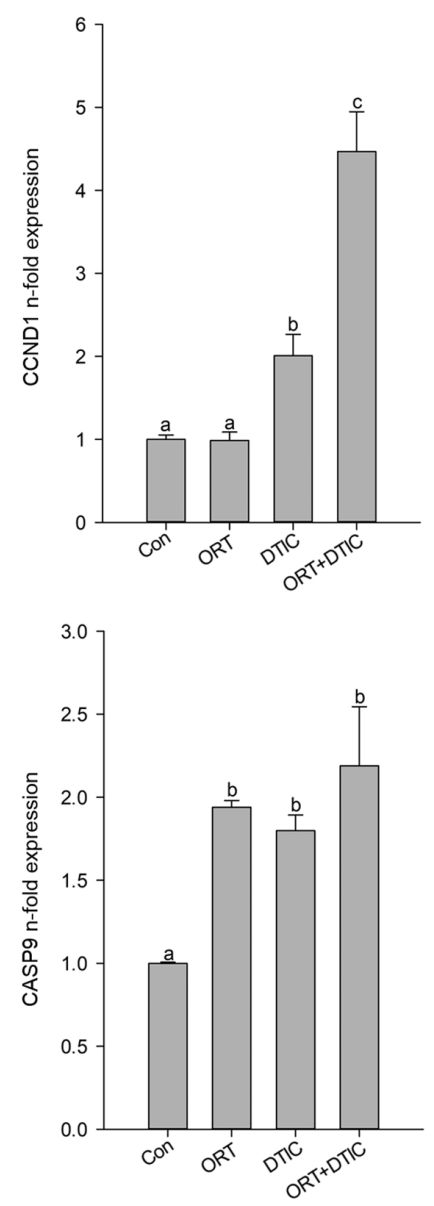
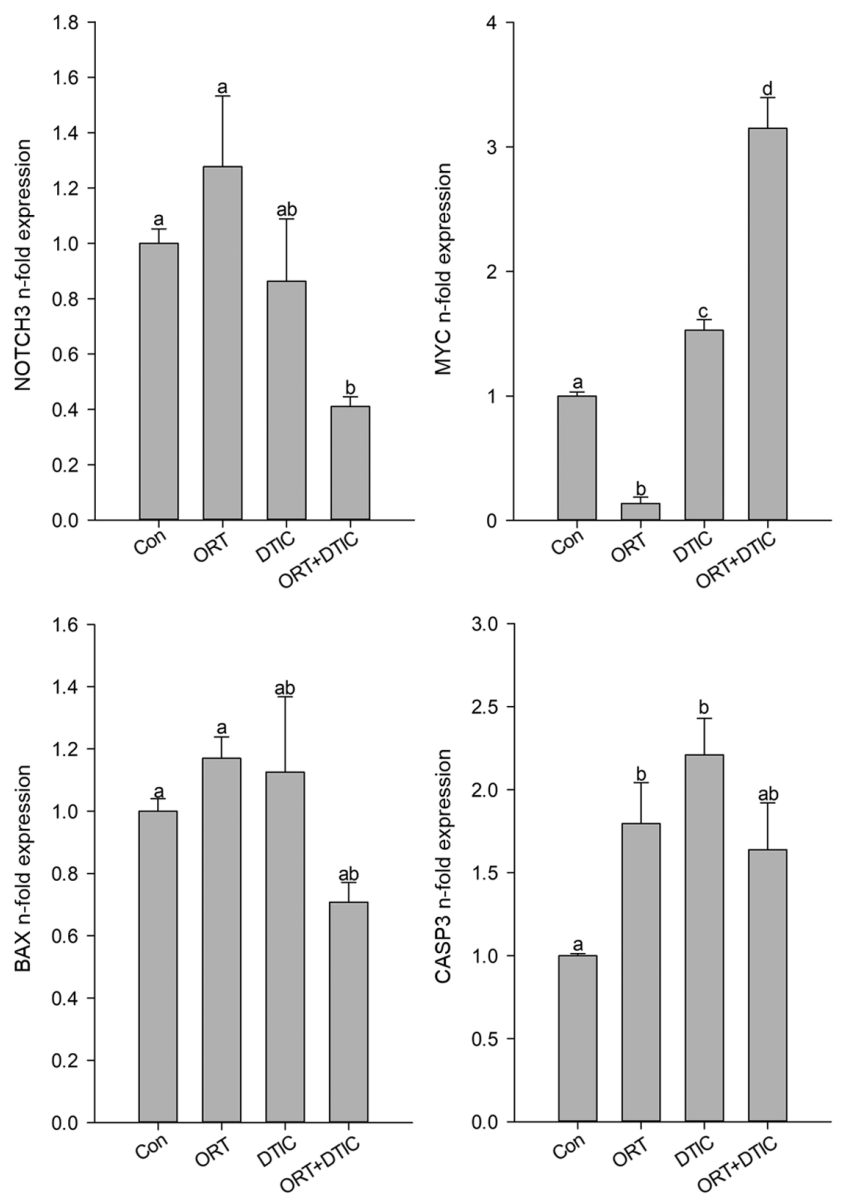

Figure 5. Protein expression levels of seven genes in the Con (untreated), ORT-treated, DTIC-treated and ORT+DTIC co-treatment groups. (A) Protein expression was analyzed using western blotting. (B) Protein expression levels of NOTCH2, NOTCH3, MYC, CCND1, BAX, CASP9 and CASP3 were normalized with those of $\beta$-actin. Values followed by the same small letter are not significantly different $(\mathrm{P}<0.05)$. ORT, oxyresveratrol; DTIC, dacarbazine; Con, control; CCND1, cyclin D1; CASP, caspase.

As shown in Fig. S3, cell migration ability seemed lower in the co-treatment group than in the ORT- and DTIC-treated groups. Although the results in Fig. S3 alone could not say that the co-treatment has an inhibitory effect on cell migration, since no quantification was performed, these results were consistent with the decreased expression of $\mathrm{NOTCH} 2$ and NOTCH3 in the co-treatment group (Fig. 5). These findings suggest that the combination of ORT and DTIC synergistically promotes $\mathrm{S}$ phase arrest by regulating the expression levels of $\mathrm{NOTCH} 2, \mathrm{NOTCH} 3, \mathrm{CCND} 1$, and MYC.
ORT reportedly promotes apoptosis by upregulating the expression of CASP3 and CASP9 (42). Additionally, ORT induces autophagy by upregulating the expression levels of LC3 I and LC3 II, which are involved in the autophagy pathway (42). As shown in Fig. 3, the proportion of cells exhibiting apoptosis was markedly higher in the co-treatment group $(23.94 \pm 3.98 \%)$ than in the ORT-treated $(15.06 \pm 1.34 \%)$ and DTIC-treated groups $(10.77 \pm 1.00 \%)$, thus implying that the combination of ORT and DTIC synergistically promoted apoptosis in WM-266-4 cells. Previous studies have revealed that decreased 


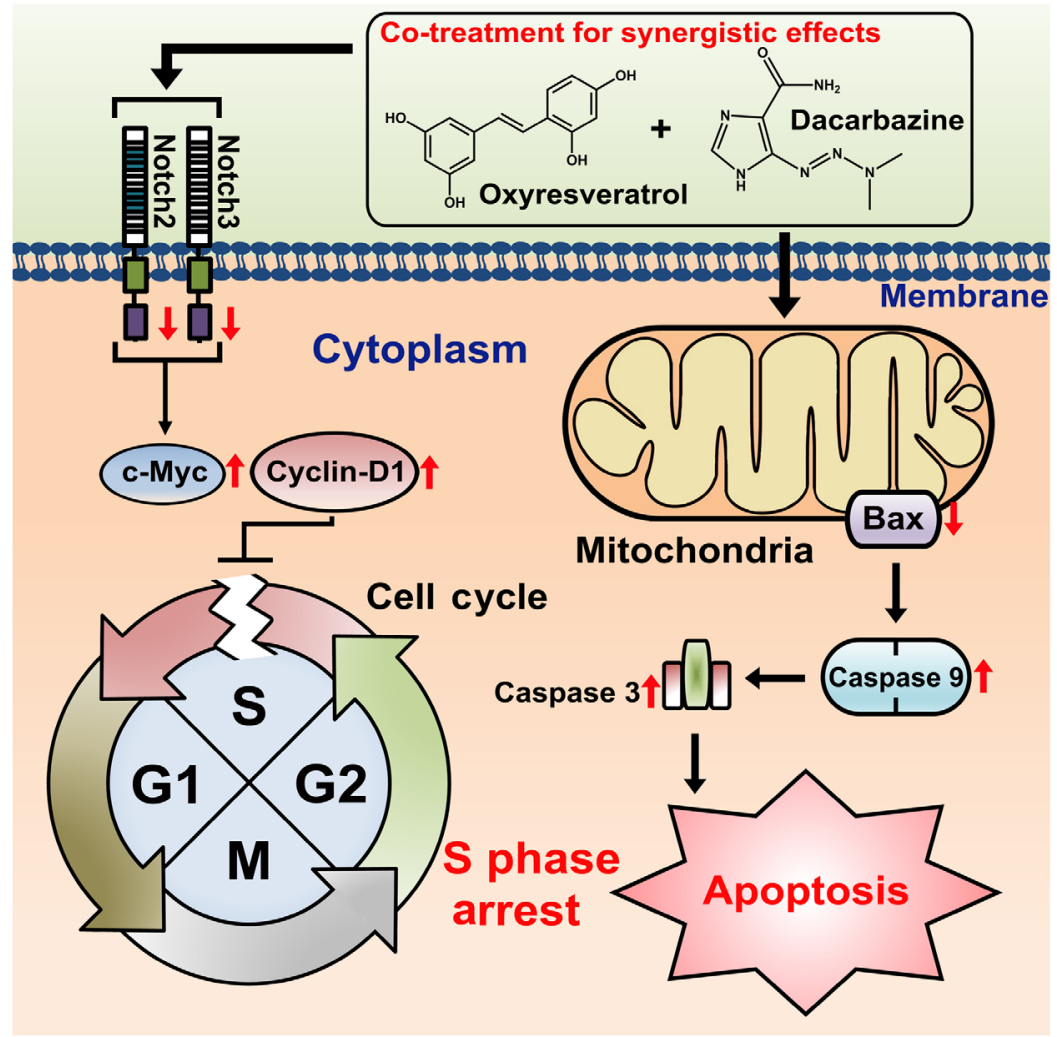

Figure 6. Proposed mechanism underlying the synergistic effects of combination treatment with ORT/DTIC on the induction of S phase arrest and apoptosis The co-treatment strategy may promote S phase arrest by downregulating the expression levels of NOTCH2 and NOTCH3, and subsequently upregulating the expression levels of MYC and CCND1. Additionally, co-treatment with ORT/DTIC may promote apoptosis by downregulating BAX expression and subsequently upregulating CASP3 and CASP9 expression. ORT, oxyresveratrol; DTIC, dacarbazine; CCND1, cyclin D1; CASP, caspase.

BAX expression and increased CASP3 and CASP9 expression can be correlated with apoptosis (26). The expression levels of BAX, CASP3, and CASP9, especially in the co-treatment group (Fig. 5), were consistent with the proportion of cells exhibiting apoptosis (Fig. 3). Therefore, the results presented in Fig. 5 may support the effects of ORT/DTIC co-treatment. The expression levels of CASP3 and CASP9 were higher in the co-treatment group than in the control group. However, co-treatment with ORT and DTIC did not synergistically upregulate the expression levels of CASP3 and CASP9, as observed following individual treatments. Thus, although treatment with ORT and DTIC enhanced CASP3 and CASP9 expression, co-treatment did not demonstrate this effect, indicating that co-treatment failed to demonstrate synergistic effects in terms of CASP3/9 expression. The cleaved forms of CASP3/9 were often employed to show the variation in expression of CASP3/9 but we had a difficulty in the detection of cleaved forms of CASP3/9 due to the lack of experiences to deal with the expressed proteins. We planned to study further the detailed signaling pathway including the cleaved forms of CASP3/9 for co-treatment with ORT and DTIC.

Herein, based on the observed results, we propose a possible mechanism underlying synergistic proliferation-inhibitory effects of the ORT/DTIC combination against WM-266-4 cells. The ORT/DTIC combination induced S phase arrest and apoptosis (Fig. 6). The treatment combination decreased the levels of NOTCH2 and NOTCH3 and consequently upregulated the expression levels of MYC and CCND1, which has been previously reported (41). Thus, the treatment combination promoted cell cycle arrest at the S phase and downregulated BAX expression in the mitochondria. The upregulated expression of CASP3 and CASP9 may promote apoptosis. This proposed mechanism is supported by results observed in a previous study demonstrating that the downregulated BAX expression results in the concomitant upregulation of CASP3 and CASP9 expression (26). The mechanism proposed in this study suggests that co-treatment with ORT and DTIC promotes apoptosis in WM-266-4 cells. Additionally, this co-treatment strategy enables the administration of lower DTIC concentrations in the presence of ORT, which exhibits low cytotoxicity $(43,44)$.

In conclusion, the findings of present study suggest that the synergistic proliferation-inhibitory effects of the ORT/DTIC combination against WM-266-4 cells can be attributed to the NOTCH signaling pathway and its downstream signaling pathways (CCND1 and MYC), as well as the BAX signaling pathway (CASP3 and CASP9). The dose of DTIC, a known cancer drug, can be reduced when co-administered with antioxidants, such as ORT, an antioxidant with low cytotoxicity.

\section{Acknowledgements}

Not applicable.

\section{Funding}

The present study was supported by a grant from the National Research Foundation of Korea (grant no. NRF-2015R1A2A2A0100650). 


\section{Availability of data and materials}

The datasets used and/or analyzed during the current study are available from the corresponding author on reasonable request.

\section{Authors' contributions}

SGL conceived the study, performed the experiments and collected the data. SGL and DGL analyzed the data. NC and YHJ assessed and confirm the authenticity of the raw data. SGL drafted the manuscript. SGL and YHJ interpreted the data. All authors have read and approved the final manuscript.

\section{Ethics approval and consent to participate}

Not applicable.

\section{Patient consent for publication}

Not applicable.

\section{Competing interests}

The authors declare that they have no competing interests.

\section{References}

1. Khoobchandani M, Ganesh N, Gabbanini S, Valgimigli L and Srivastava MM: Phytochemical potential of Eruca sativa for inhibition of melanoma tumor growth. Fitoterapia 82: 647-653, 2011.

2. Siegel RL, Miller KD and Jemal A: Cancer statistics, 2020. CA Cancer J Clin 70: 7-30, 2020.

3. Siegel RL, Miller KD and Jemal A: Cancer statistics, 2015. CA Cancer J Clin 65: 5-29, 2015.

4. Yu B, Wang Y, Yu X, Zhang H, Zhu J, Wang C, Chen F, Liu C, Wang $\mathrm{J}$ and Zhu H: Cuprous oxide nanoparticle-inhibited melanoma progress by targeting melanoma stem cells. Int J Nanomedicine 12: 2553-2567, 2017.

5. Ma C and Armstrong AW: Severe adverse events from the treatment of advanced melanoma: A systematic review of severe side effects associated with ipilimumab, vemurafenib, interferon alfa-2b, dacarbazine and interleukin-2. J Dermatolog Treat 25: 401-408, 2014

6. Lee SG and Chae YS: New systemic treatment for malignant melanoma. Korean J Intern Med (Korean Assoc Intern Med) 85 357-363, 2013.

7. Baharara J, Amini E, Nikdel N and Salek-Abdollahi F: The cytotoxicity of dacarbazine potentiated by sea cucumber saponin in resistant B16F10 melanoma cells through apoptosis induction. Avicenna J Med Biotechnol 8: 112-119, 2016.

8. Mongolsuk S, Robertson A and Towers R: 2: 4: 3': 5'-Tetrahydroxystilbene from Artocarpus lakoocha. J Chem Soc 429: 2231-2233, 1957.

9. Tan Y, Liu C and Chen R: Phenolic constituents from stem bark of Morus wittiorum and their anti-inflammation and cytotoxicity. Zhongguo Zhongyao Zazhi 35: 2700-2703, 2010 (In Chinese).

10. Choi SM, Kim DH, Chun KS and Choi JS: Carnosol induces apoptotic cell death through ROS-dependent inactivation of STAT3 in human melanoma G361 cells. Appl Biol Chem 62: $1-11,2019$.

11. Kim YM, Yun J, Lee C-K, Lee H, Min KR and Kim Y: Oxyresveratrol and hydroxystilbene compounds. Inhibitory effect on tyrosinase and mechanism of action. J Biol Chem 277: 16340-16344, 2002.

12. Chung KO, Kim BY, Lee MH, Kim YR, Chung HY, Park JH and Moon JO: In-vitro and in-vivo anti-inflammatory effect of oxyresveratrol from Morus alba L. J Pharm Pharmacol 55: 1695-1700, 2003.
13. Qiu F, Komatsu K, Saito K, Kawasaki K, Yao X and Kano Y; Pharmacokinetic study of mulberroside A and its metabolites in rat: Pharmacological properties of traditional medicines. XXII. Pharmacokinetic study of mulberroside A and its metabolites in rat. Biol Pharm Bull 19: 1463-1467, 1996.

14. Kim J, Cho N, Kim EM, Park KS, Kang YW, Nam JH, Nam MS and Kim KK: Cudrania tricuspidata leaf extracts and its components, chlorogenic acid, kaempferol, and quercetin, increase claudin 1 expression in human keratinocytes, enhancing intercellular tight junction capacity. Appl Biol Chem 63: 1-9, 2020.

15. McDermott DF, Mier JW, Lawrence DP, van den Brink MR, Clancy MA, Rubin KM and Atkins MB: A phase II pilot trial of concurrent biochemotherapy with cisplatin, vinblastine, dacarbazine, interleukin 2 , and interferon $\alpha-2 B$ in patients with metastatic melanoma. Clin Cancer Res 6: 2201-2208, 2000.

16. Cocconi G, Bella M, Calabresi F, Tonato M, Canaletti R, Boni C, Buzzi F, Ceci G, Corgna E, Costa P, et al: Treatment of metastatic malignant melanoma with dacarbazine plus tamoxifen. N Engl J Med 327: 516-523, 1992.

17. Bedikian AY, Millward M, Pehamberger H, Conry R, Gore M, Trefzer U, Pavlick AC, DeConti R, Hersh EM, Hersey P, et al; Oblimersen Melanoma Study Group: Bcl-2 antisense (oblimersen sodium) plus dacarbazine in patients with advanced melanoma: The Oblimersen Melanoma Study Group. J Clin Oncol 24: 4738-4745, 2006.

18. Chen H-M, Muramoto K, Yamauchi F, Fujimoto K and Nokihara K: Antioxidative properties of histidine-containing peptides designed from peptide fragments found in the digests of a soybean protein. J Agric Food Chem 46: 49-53, 1998.

19. Monzani E, Facchetti F, Galmozzi E, Corsini E, Benetti A, Cavazzin C, Gritti A, Piccinini A, Porro D, Santinami M, et al: Melanoma contains CD133 and ABCG2 positive cells with enhanced tumourigenic potential. Eur J Cancer 43: 935-946, 2007.

20. Utikal J, Leiter U, Udart M, Kaskel P, Peter RU and Krähn GM: Expression of c-myc and bcl-2 in primary and advanced cutaneous melanoma. Cancer Invest 20: 914-921, 2002.

21. Choi HJ, Yee S-B, Park SE, Im E, Jung JH, Chung HY, Choi YH and Kim ND: Petrotetrayndiol A induces cell cycle arrest and apoptosis in SK-MEL-2 human melanoma cells through cytochrome c-mediated activation of caspases. Cancer Lett 232: 214-225, 2006

22. Shanehbandi D, Zarredar H, Asadi M, Zafari V, Esmaeili S, Seyedrezazadeh E, Soleimani Z, Jadid HS, Eyvazi S, Feyziniya S, et al: Anticancer impacts of Terminalia catappa extract on SW480 colorectal neoplasm cell line. J Gastrointest Cancer 50: 1-7, 2019.

23. Orangi M, Pasdaran A, Shanehbandi D, Kazemi T, Yousefi B, Hosseini BA and Baradaran B: Kazemi T, Yousefi B, Hosseini BA and Baradran B: Cytotoxic and apoptotic activities of methanolic subfractions of Scrophularia oxysepala against human breast cancer cell line. Evid Based Complement Alternat Med 2016: 8540640, 2016.

24. Xu X, Liu Y, Wang L, He J, Zhang H, Chen X, Li Y, Yang J and Tao J: Gambogic acid induces apoptosis by regulating the expression of Bax and Bcl-2 and enhancing caspase-3 activity in human malignant melanoma A375 cells. Int J Dermatol 48: 186-192, 2009.

25. Livak KJ and Schmittgen TD: Analysis of relative gene expression data using real-time quantitative PCR and the 2(-Delta Delta C(T)) Method. Methods 25: 402-408, 2001.

26. Yadav V, Varshney P, Sultana S, Yadav J and Saini N: Moxifloxacin and ciprofloxacin induces S-phase arrest and augments apoptotic effects of cisplatin in human pancreatic cancer cells via ERK activation. BMC Cancer 15: 581, 2015.

27. Hu S, Zheng Z, Zhang X, Chen F and Wang M: Oxyresveratrol and trans-dihydromorin from the twigs of Cudrania tricuspidata as hypopigmenting agents against melanogenesis. J Funct Foods 13: 375-383, 2015.

28. Hu S, Chen F and Wang M: Photoprotective effects of oxyresveratrol and Kuwanon O on DNA damage induced by UVA in human epidermal keratinocytes. Chem Res Toxicol 28: 541-548, 2015.

29. Bougatef A, Hajji M, Balti R, Lassoued I, Triki-Ellouz Y and Nasri M: Antioxidant and free radical-scavenging activities of smooth hound (Mustelus mustelus) muscle protein hydrolysates obtained by gastrointestinal proteases. Food Chem 114: 1198-1205, 2009.

30. Lim YH, Kim KH and Kim JK: Source, biosynthesis, biological activities and pharmacokinetics of oxyresveratrol. Korean J Food Sci Technol 47: 545-555, 2015. 
31. Strassmann G, Masui Y, Chizzonite R and Fong M: Mechanisms of experimental cancer cachexia. Local involvement of IL-1 in colon-26 tumor. J Immunol 150: 2341-2345, 1993.

32. Takagi N, Mihara M, Moriya Y, Nishimoto N, Yoshizaki K, Kishimoto T, Takeda Y and Ohsugi Y: Blockage of interleukin-6 receptor ameliorates joint disease in murine collagen-induced arthritis. Arthritis Rheum 41: 2117-2121, 1998.

33. Zhang XG, Bataille R, Jourdan M, Saeland S, Banchereau J, Mannoni P and Klein B: Granulocyte-macrophage colony-stimulating factor synergizes with interleukin-6 in supporting the proliferation of human myeloma cells. Blood 76: 2599-2605, 1990.

34. Lee AK, Sung SH, Kim YC and Kim SG: Inhibition of lipopolysaccharide-inducible nitric oxide synthase, TNF- $\alpha$ and COX- 2 expression by sauchinone effects on I-kappaBalpha phosphorylation, C/EBP and AP-1 activation. Br J Pharmacol 139: 11-20, 2003.

35. Choi EM and Hwang JK: Effects of Morus alba leaf extract on the production of nitric oxide, prostaglandin E2 and cytokines in RAW264.7 macrophages. Fitoterapia 76: 608-613, 2005.

36. Naserian M, Ramazani E, Iranshahi M and Tayarani-Najaran Z: The role of SAPK/JNK pathway in the synergistic effects of metformin and dacarbazine on apoptosis in Raji and Ramos lymphoma cells. Curr Mol Pharmacol 11: 336-342, 2018.

37. Pitot HC and Sirica AE: The stages of initiation and promotion in hepatocarcinogenesis. Biochim Biophys Acta 605: 191-215, 1980.

38. Koprowska K, Hartman ML, Sztiller-Sikorska M and Czyz ME: Parthenolide enhances dacarbazine activity against melanoma cells. Anticancer Drugs 24: 835-845, 2013.
39. Prall OW, Sarcevic B, Musgrove EA, Watts CK and Sutherland RL: Estrogen-induced activation of Cdk4 and Cdk2 during G1-S phase progression is accompanied by increased cyclin D1 expression and decreased cyclin-dependent kinase inhibitor association with cyclin E-Cdk2. J Biol Chem 272: 10882-10894, 1997.

40. Sheen J-H and Dickson RB: Overexpression of c-Myc alters $\mathrm{G}(1) / \mathrm{S}$ arrest following ionizing radiation. Mol Cell Biol 22: 1819-1833, 2002

41. Zhao WX, Wu ZM, Liu W and Lin JH: Notch2 and Notch3 suppress the proliferation and mediate invasion of trophoblast cell lines. Biol Open 6: 1123-1129, 2017.

42. Rahman MA, Bishayee K, Sadra A and Huh SO: Oxyresveratrol activates parallel apoptotic and autophagic cell death pathways in neuroblastoma cells. Biochim Biophys Acta, Gen Subj 1861: 23-36, 2017.

43. Lorenz P, Roychowdhury S, Engelmann M, Wolf G and Horn TF: Oxyresveratrol and resveratrol are potent antioxidants and free radical scavengers: Effect on nitrosative and oxidative stress derived from microglial cells. Nitric Oxide 9: 64-76, 2003.

44. Kim SH and Chung SH: Comparison of high dose interferon- $\alpha 2 b$ immunotherapy and dacarbazine chemotherapy as postoperative treatment of malignant melanoma. J Korean Orthop Assoc 51: 426-431, 2016.

This work is licensed under a Creative Commons Attribution-NonCommercial-NoDerivatives 4.0 International (CC BY-NC-ND 4.0) License. 